\title{
Abundancia relativa y dieta de Grundulus bogotensis (Characiformes: Characidae) en el altiplano Cundiboyacense, Colombia
}

\author{
Camilo Andrés Roa-Fuentes ${ }^{\boxplus 1}$; Saúl Prada-Pedreros ${ }^{1}$; Ricardo Álvarez-Zamora ${ }^{1}$; Carlos A. \\ Rivera Rondón ${ }^{2}$; Javier A. Maldonado-Ocampo ${ }^{1}$
}

Received: 11-09-2012 Accepted: 15-02-2013 Published on line: 28-03-2013 Edited by Alberto Acosta

Relative abundance and diet of Grundulus bogotensis (Characiformes: Characidae) in the Cundiboyacense plateau, Colombia. Abstract. To compare the relative abundance of Grundulus bogotensis in two river basins and the diet of the populations of three basins in the Cundiboyacense plateau, we sampled 10 localities using electrofishing equipment between March and June 2006. The relative abundance in each locality was expressed as the number of fish caught per hour on a $100 \mathrm{~m}$ stretch; any significant differences in relative abundance among basins were identified via a Kruskal-Wallis test. To quantify the diet, we used the volumetric method. Similarities between the basins were determined using three multivariate analyzes: nonmetric multidimensional scaling, analysis of similarity and similarity percentages. In the end, we collected a total of 675 individuals during 600 minutes of capture effort. The abundance of this species in the Bogotá river basin was significantly lower compared to that of the Suárez basin. The dietary composition, of autochthonous origin, primarily immature insects and microcrustaceans and the volume of items proved similar between the basins. In conclusion, the dietary analysis indicated a similarity exceeding $60 \%$ in the feed resource use for the three basins. The relative abundance of populations of $G$. bogotensis in the Bogotá river basin was lower than in the Suárez river basin, the latter having, possibly, the greatest abundance of this species throughout its range. The data suggests the most favorable conditions for the survival of the species in Lake Tota.

Keywords: Guapucha, Bogotá river, Suárez river, Tota lake, threatened species

1 Laboratorio de Ictiología, Unidad de Ecología y Sistemática -UNESIS-, Departamento de Biología, Facultad de Ciencias, Pontificia Universidad Javeriana, Carrera 7 N 40-62, Bogotá D.C., Colombia.

2 Laboratorio de Limnología, Unidad de Ecología y Sistemática -UNESIS-, Departamento de Biología, Facultad de Ciencias, Pontificia Universidad Javeriana, Cra. 7 No 40-62. Bogotá, Colombia.
Resumen. Se comparó la abundancia relativa de Grundulus bogotensis entre dos cuencas y la dieta entre poblaciones de tres cuencas del altiplano Cundiboyacense. Entre marzo y junio de 2006 se realizaron muestreos en 10 localidades utilizando un equipo de electropesca. La abundancia relativa de cada localidad se expresó como el número de individuos capturado por hora en un tramo de $100 \mathrm{~m}$. Se utilizó un test de Kruskal-Wallis para determinar diferencias significativas en la abundancia relativa entre cuencas. Para cuantificar la dieta se utilizó el método volumétrico y para determinar el grado de similaridad entre cuencas se recurrió a tres análisis multivariados: análisis de escalonamiento multidimensional no métrico (NMDS), análisis de similaridad (ANOSIM) y análisis de porcentaje de similaridad (SIMPER). Se capturaron en total 675 individuos, empleando 600 minutos de esfuerzo de captura. La abundancia de la especie en la cuenca del río Bogotá fue significativamente menor respecto a la del río Suárez. La composición de la dieta y volumen de ítems entre cuencas fue similar. El material consumido fue de origen autóctono y los ítems más importantes fueron los insectos inmaduros y los microcrustáceos. La abundancia relativa de las poblaciones de $G$. bogotensis en la cuenca del río Bogotá fue menor que en la cuenca del río Suárez. La cuenca del río Suárez probablemente soporta la mayor abundancia de la especie en todo su rango de distribución y los datos sugieren condiciones favorables para la sobrevivencia de la Guapucha en el lago de Tota. El análisis de la dieta indicó una similaridad mayor al $60 \%$ en la utilización del recurso alimentar en las tres cuencas.

Resumo. Comparar a abundância relativa de Grundulus bogotensis entre duas bacias e a dieta entre populações de três bacias do planalto Cundiboyacense. Entre março e junho de 2006 foram realizadas coletas em 10 localidades utilizando equipamento de pesca elétrica. A abundância relativa da espécie em cada localidade foi expressa como o número de indivíduos capturados por hora em um trecho de $100 \mathrm{~m}$ de extensão. Foi utilizado o teste de Kruskal-Wallis para determinar diferenças significativas na abundância relativa entre as bacias. Para quantificar a dieta foi utilizado o método volumétrico e para determinar o grau de similaridade entre bacias foram utilizadas três análises multivariadas: análise de escalonamento multidimensional não métrico (NMDS), análise de similaridade (ANOSIM) e análise de porcentagem de similaridade (SIMPER). No total foram capturados 675 indivíduos, empregando 600 minutos de esforço de captura. A abundância desta espécie na bacia do rio Bogotá foi significativamente menor que na bacia do rio Suárez. A composição da dieta e volume dos itens de alimento entre bacias foram similares. Os recursos consumidos foram de origem autóctone e os itens mais abundantes foram insetos imaturos e microcrustáceos. A abundância relativa das populações de $G$ bogotensis na bacia do rio Bogotá foi menor do que na bacia do rio Suárez. A bacia do rio Suárez provavelmente suporta a maior abundância da espécie em toda a área de distribuição e os dados sugerem condições favoráveis para a sobrevivência da Guapucha no Lago de Tota. A análise da dieta indicou uma similaridade maior a $60 \%$ na utilização do recurso alimentar nas três bacias. 


\section{Introducción}

La interacción entre el ambiente y los procesos poblacionales afecta los patrones de distribución y abundancia de las especies en los ecosistemas (Brown 1984). Para una especie determinada, ciertas localidades son más favorables ya que la combinación de variables ambientales cumple los requerimientos de nicho; de igual manera, existirán otras localidades donde la abundancia será menor debido a que los recursos son escasos o las condiciones superan los límites que pueden ser tolerados por dicha especie (Brown 1984). En los sistemas acuáticos existen diversos factores ambientales naturales (tamaño del río, régimen de flujo) que afectan de manera importante la estructura del hábitat, distribución y abundancia de las especies (Gorman y Karr 1978, Tejerina-Garro et al. 2005). Sin embargo, en las últimas décadas los cuerpos de agua han sufrido cambios profundos de origen antrópico alterando los factores naturales que influyen sobre la biota $y$ amenazan a la biodiversidad acuática (Agostinho et al. 2005, Kruk 2007).

La pérdida de especies o alteración de la estructura de comunidades y poblaciones en sistemas acuáticos están relacionadas con ambientes contaminados y eutrofizados, degradación de hábitat e introducción de especies particularmente en regiones con alta densidad de población humana (Collares-Pereira et al. 2000, Agostinho et al. 2005). 'Tradicionalmente se asume que la pérdida de especies está acompañada por un cambio en la distribución de las abundancias desde una serie log normal para una geométrica (i.e., en ambientes no perturbados la abundancia de las especies se distribuye de manera uniforme pero una vez modificados son pobres en especies y exhiben alta dominancia) (Magurran y Phillip 2001). Por otro lado, la disponibilidad y tipo de recurso para alimento también depende de las características de cada ambiente. En sistemas lénticos, por ejemplo, niveles elevados de sedimentación y alta penetración de la luz facilitan la localización de presas pequeñas (ejemplo: cladóceros y copépodos) por parte de los peces y los ítems consumidos son en su mayoría de origen autóctono (Albrecht y Caramaschi 2003). También se han observado cambios en los tipos de ítems consumidos por la ictiofauna en función del nivel de degradación de los sistemas acuáticos
(Rocha et al. 2009).

La Guapucha, Grundulus bogotensis (Humboldt 1821), es un pez endémico del altiplano Cundiboyacense, Colombia (Fowler 1942). Se distribuye en la Sabana de Bogotá y hacia el norte hasta el departamento de Santander (Dahl 1971, Miles 1971). El área de distribución natural de la especie estaba restringida a menos de 30.000 $\mathrm{km}^{2}$ (Álvarez-León et al. 2002), sin embargo, ha sido ampliada debido al trasplante en la década de los 50's en diferentes cuerpos de agua, entre ellos el lago de Tota en el departamento de Boyacá (Alvarado y Gutiérrez 2002). En general, los sistemas que habita presentan un avanzado proceso de deterioro ambiental, destacándose la cuenca del río Bogotá por ser la más degradada de Colombia (CAR 2002). Los procesos de deterioro ambiental están asociados al desarrollo agrícola e industrial, inadecuado manejo de los residuos generados por municipios adyacentes, desecación de humedales, actividad minera (Álvarez-León et al. 2002, CAR 2002, Álvarez-León et al. 2012) y urbanización.

En la primera edición del libro rojo de peces de agua dulce de Colombia (Mojica et al. 2002), G. bogotensis se incluyó en la categoría nacional "casi amenazada", no obstante en la segunda edición (Mojica et al. 2012), su estatus cambió siendo una de las tres especies en la categoría "preocupación menor". Entre las medidas para su protección se han recomendado el desarrollo de estudios que fortalezcan y aporten conocimiento sobre su biología y ecología (Álvarez-León et al. 2002, Álvarez-León et al. 2012), ya que este tipo de información es clave para elaborar propuestas para la gestión apropiada de los recursos, determinar el papel de las especies en los sistemas acuáticos, y en consecuencia, para mantener el equilibrio dentro de un ecosistema (Lobón-Cerviá 1991, Lizama y Ambrósio 2002).

Los estudios que abordan aspectos bioecológicos de $G$. bogotensis en su mayoría se encuadran en la denominada 'literatura gris', y son pocos los trabajos publicados sobre la ecología (Mora et al. 1992, Pantoja-Agreda y Pantoja-Agreda 2003, Rivera-Rondón et al. 2008, Pinzón-González y Prada-Pedreros 2011), morfología (León et al. 2005), información genética (González-Acosta et al. 1992) y prácticamente nulos los relacionados con la distribución en el altiplano y abundancia 
relativa de esta especie. De acuerdo con esto y con la necesidad de contar con datos sobre la distribución y autoecología de la Guapucha, los objetivos de este estudio fueron: 1) comparar la abundancia relativa de $G$. bogotensis entre poblaciones de las cuencas del río Bogotá, río Suárez y lago de Tota y 2) determinar si existen diferencias en la dieta de $G$. bogotensis entre las cuencas estudiadas. Se esperaba que la abundancia relativa en las poblaciones de la cuenca del río Bogotá fuese menor debido al alto grado de degradación que presenta esta cuenca y dadas las características de los ambientes estudiados, que la dieta de los individuos fuese semejante en las tres cuencas.

\section{Materiales y métodos}

Área de estudio: El altiplano Cundiboyacense está situado entre los departamentos de Cundinamarca y Boyacá, su extensión aproximada es de 19.529 km² y geográficamente se encuentra entre los $3^{\circ} 45^{\prime} 13^{\prime \prime}$ y los 6 03 '13" N y entre los 72³9'40" y los 74 $52^{\circ} 30^{\prime}$ " W (IGAC 1984). Los principales ríos que drenan por ésta región son: Sumapaz, Bogotá, Seco, Negro, Minero, Ubaté y Suárez; los ríos que drenan hacia los llanos orientales, incluyendo cuenca de Tota, alimentan la cuenca del río Meta (CAR 2002). La altitud en el altiplano Cundiboyacense oscila entre $2600 \mathrm{~m}$ en la zona plana hasta $3800 \mathrm{~m}$ en el caso de los cerros que la circundan; en el sector drenado por los ríos Bogotá y Suárez, se registran temperaturas medias entre los 12 y $14{ }^{\circ} \mathrm{C}$ que descienden en el sector suroriental de Bogotá, sobre el páramo de Sumapáz, alcanzando valores inferiores a $10{ }^{\circ} \mathrm{C}$ (CAR 2002) (Figura 1).

Sitios de captura: Los sitios de captura se seleccionaron con ayuda de un mapa del altiplano Cundiboyacense escala 1:400.000. Con la selección de las localidades se buscó obtener información de la mayor varianza posible, entre más diferentes fueran los hábitats mayor el grado de información sobre el comportamiento de la varianza. En campo, la localización exacta del punto de muestreo (latitud, longitud y altitud) se georreferenció con ayuda de una unidad de GPS marca Garmin, modelo GPS 72. Se realizaron muestreos en 10 localidades,

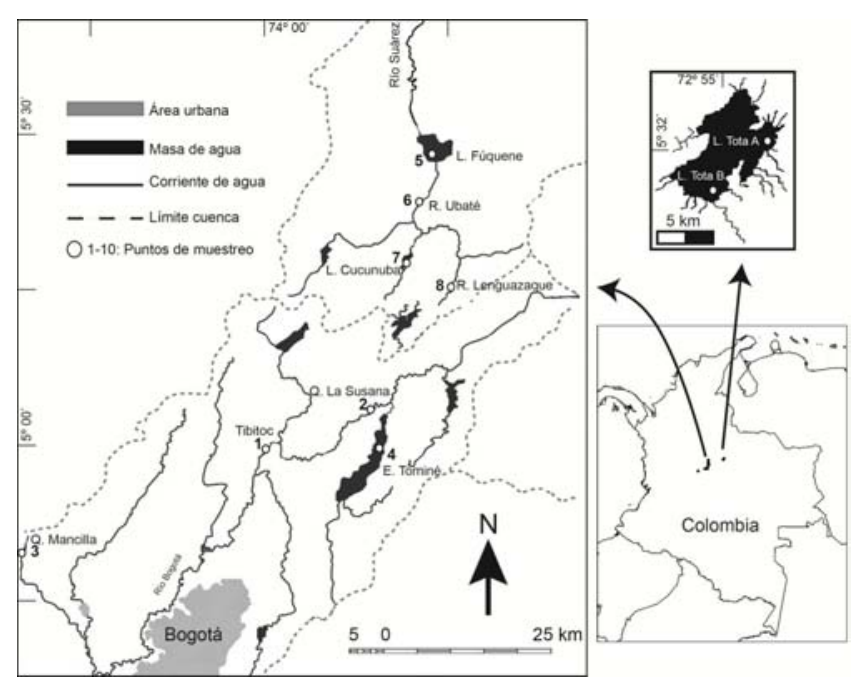

Fig. 1. Localización de los sitios de muestreo en el altiplano Cundiboyacense, Colombia. 1-Tibitoc; 2-Quebrada La Susana; 3-Quebrada Mancilla; 4-Embalse de Tominé; 5-Laguna de Fúquene; 6-Río Ubaté; 7-Laguna de Cucunubá; 8-Río Lenguazaque; 9-Lago de Tota A; 10Lago de Tota B.

distribuidas por cuenca hidrológica de la siguiente manera: cuatro pertenecientes al río Bogotá, cuatro al río Suárez y dos al lago de Tota (Figura 1). En cada sitio de muestreo se realizó una caracterización visual de las macrófitas acuáticas presentes y otra fisicoquímica midiendo las siguientes variables: temperatura, oxígeno disuelto, $\mathrm{pH}$ y conductividad. Para esto se utilizó un potenciómetro y un oxímetro ambos de marca Oakton (Tabla 1).

Captura y abundancia relativa: Entre marzo y junio de 2006 se realizaron muestreos en las localidades seleccionadas utilizando un equipo de pesca eléctrica. Los muestreos se estandarizaron tanto en espacio como en tiempo; así, la abundancia por localidad corresponde al número de peces capturados en un tramo de $100 \mathrm{~m}$ durante una hora de electropesca (CPUE). Ya que las distancias y tiempos de muestreo fueron equivalentes y en los lagos se realizó el muestreo en área de baja profundidad se asume que la eficiencia de colecta del equipo fue semejante en todos los puntos de muestreo.

En estudios poblacionales de peces, la captura por unidad de esfuerzo (CPUE) se puede utilizar como un índice de abundancia (Battaile y Quinn 
Tabla 1. Ubicación geográfica de las localidades de muestreo en el altiplano Cundiboyacense, macrófitas acuáticas presentes y valores de temperatura, oxígeno disuelto (OD), $\mathrm{pH}$ y conductividad del agua durante la colecta.

\begin{tabular}{|c|c|c|c|c|c|c|c|c|}
\hline Cuenca & Localidad & $\begin{array}{l}\text { Macrófita } \\
\text { acuática }\end{array}$ & $\begin{array}{l}\text { Posición } \\
\text { geográfica }\end{array}$ & $\begin{array}{c}\text { Altura } \\
\text { (m.s.n.m.) }\end{array}$ & $\begin{array}{l}\text { Temperatura } \\
\text { del agua }\left({ }^{\circ} \mathrm{C}\right)\end{array}$ & $\begin{array}{c}\text { OD } \\
(\mathrm{mg} / \mathrm{L})\end{array}$ & $\mathrm{pH}$ & $\begin{array}{c}\text { Conductividad } \\
(\mu \mathrm{S} / \mathrm{cm})\end{array}$ \\
\hline \multirow[t]{4}{*}{ Bogotá } & Río Bogotá (Tibitoc) & Eichhornia sp. & $\begin{array}{l}4^{\circ} 58^{\prime} 38,2^{\prime \prime} \mathrm{N} \\
73^{\circ} 58^{\prime} 01^{\prime \prime W}\end{array}$ & 2563 & 16,6 & 2,0 & 6,7 & 115,9 \\
\hline & Quebrada La Susana & Poaceae & $\begin{array}{c}5^{\circ} 03^{\prime} 50,3^{\prime \prime} \mathrm{N} \\
73^{\circ} 49^{\prime} 51,8^{\prime \prime} \mathrm{W}\end{array}$ & 2573 & 17,3 & 2,6 & 6,8 & 133,3 \\
\hline & Quebrada Mancilla & Poaceae & $\begin{array}{l}4^{\circ} 51^{\prime} 09,7^{\prime \prime N} \\
74^{\circ} 21^{\prime} 01,8^{\prime \prime} \mathrm{W}\end{array}$ & 2582 & 14,0 & 7,6 & 6,6 & 36,3 \\
\hline & Embalse de Tominé & Eichhornia sp. & $\begin{array}{l}4^{\circ} 59^{\prime} 10,7^{\prime \prime} \mathrm{N} \\
73^{\circ} 49^{\prime} 09^{\prime \prime} \mathrm{W}\end{array}$ & 2622 & 17,6 & 6,4 & 6,6 & 36,1 \\
\hline \multirow[t]{4}{*}{ Suárez } & Laguna de Fúquene & $\begin{array}{c}\text { Eichbornia sp., } \\
\text { Schoenoplectus } \\
\text { californicus, } \\
\text { Egeria sp. }\end{array}$ & $\begin{array}{c}5^{\circ} 28^{\prime} 05,4^{\prime \prime} \mathrm{N} \\
73^{\circ} 46^{\prime} 20,7^{\prime \prime W}\end{array}$ & 2551 & 21,5 & 6,0 & 6,2 & 136,5 \\
\hline & Río Ubaté & Eichhornia sp. & $\begin{array}{c}5^{\circ} 23^{\prime} 00,8^{\prime \prime} \mathrm{N} \\
73^{\circ} 45^{\prime} 53,9^{\prime \prime W}\end{array}$ & 2559 & 16,4 & 2,9 & 6,3 & 189,1 \\
\hline & Laguna de Cucunubá & Egeria sp. & $\begin{array}{c}5^{\circ} 17^{\prime} 01,4^{\prime \prime N} \\
73^{\circ} 47^{\prime} 11,9^{\prime \prime W}\end{array}$ & 2563 & 19,3 & 4,1 & 6,8 & 1005,5 \\
\hline & Río Lenguazaque & Poaceae & $\begin{array}{c}5^{\circ} 12^{\prime} 08,9^{\prime \prime} \mathrm{N} \\
73^{\circ} 44^{\prime} 38,7^{\prime \prime W}\end{array}$ & 2842 & 19,8 & 6,4 & 6,8 & 35,9 \\
\hline \multirow[t]{2}{*}{ Tota } & Lago de Tota A & $\begin{array}{c}\text { Schoenoplectus } \\
\text { californicus, } \\
\text { Egeria sp. }\end{array}$ & $\begin{array}{c}5^{\circ} 33^{\prime} 35,5^{\prime \prime} \mathrm{N} \\
72^{\circ} 53^{\prime} 08,8^{\prime \prime} \mathrm{W}\end{array}$ & 3037 & 13,8 & 6,6 & 6,7 & 69,8 \\
\hline & Lago de Tota B & $\begin{array}{c}\text { Schoenoplectus } \\
\text { sp. }\end{array}$ & $\begin{array}{l}5^{\circ} 29^{\prime} 29^{\prime \prime} \mathrm{N} \\
72^{\circ} 56^{\prime} 1 " \mathrm{~W}\end{array}$ & 3042 & 15,8 & 6,8 & 7,3 & 73,6 \\
\hline
\end{tabular}

2004, Haggarty y King 2006). Aunque la CPUE no necesariamente es un índice preciso (Battaile y Quinn 2004, Harley et al. 2001), su estandarización (por ejemplo: duración de pesca y equipos predeterminados) permite que las variaciones que no están relacionadas a la abundancia (hiperestabilidad, hiperagregación, capturabilidad) sean relativamente bien controladas (Battaile y Quinn 2004, Haggarty y King 2006). Por lo tanto, es posible utilizar de manera efectiva a la CPUE para monitorear las poblaciones de peces, permitiendo determinar si las abundancias incrementan, disminuyen o permanecen constantes
(Haggarty y King 2006).

En campo, los peces se fijaron en una solución de formaldehído al 10\% y finalmente se conservaron en etanol al 70\%, siendo depositados en la Colección de Peces del Museo de Historia Natural de la Pontificia Universidad Javeriana (MPUJ) Bogotá, Colombia. Para estimar si existen diferencias significativas en la CPUE entre las cuencas hidrográficas Suárez y Bogotá se utilizó una prueba de Kruskall-Wallis empleando el software Statistica v7 (StatSoft 2004). No se realizó una comparación estadística con el lago de Tota debido a su bajo número de puntos de muestreo. 
Dieta: En ocho de las 10 localidades se examinaron 30 estómagos por localidad y en las dos restantes, Tibitoc y quebrada Mancilla, 12 y 15 estómagos respectivamente, para un total de 267 estómagos analizados, todos con contenido estomacal y provenientes de individuos adultos de tamaño similar para evitar el efecto del tamaño en los análisis de la dieta. Se extrajeron las vísceras de los peces y el contenido estomacal fue examinado bajo estereomicroscopio. Con la ayuda de una cámara volumétrica se obtuvo el volumen de los ítems alimentares que posteriormente se agruparon de acuerdo al grupo taxonómico y su origen de la siguiente manera: insectos de origen autóctono (Coleoptera, Diptera, Hemiptera, Odonata, Trichoptera), insectos de origen alóctono (Hymenoptera), arácnidos de origen alóctono (Acari terrestres), crustáceos (Amphipoda, Cladocera, Copepoda, Ostracoda), moluscos (Physidae, Bivalvia), nemátodos, macrófitas (semillas, raíces, fragmentos de plantas), detrito (materia orgánica particulada) y escamas. Los ítems se identificaron utilizando bibliografía especializada (McCafferty y Provonsha 1981, Merritt y Cummins 1981, Roldán 1996).

Previo a los análisis de similaridad de la dieta entre cuencas, los ítems de alimento agrupados fueron: 1) estandarizados (cada grupo de ítems se expresó como porcentaje del volumen total de la localidad) para reducir el efecto del volumen estomacal examinado en cada localidad y 2) transformados (raíz cuadrada) para disminuir la influencia de las categorías alimentares dominantes. Para el cálculo de las matrices de similaridad se utilizó el coeficiente de Bray-Curtis. Con el fin de determinar si existen diferencias en la composición de la dieta de $G$. bogotensis entre las tres cuencas, se utilizaron los siguientes análisis multivariados: análisis de escalonamiento multidimensional no métrico (NMDS) con sobreposición de cluster (UPGMA), análisis de similaridad (ANOSIM, 999 permutaciones) y análisis de porcentaje de similaridad (SIMPER). Para los análisis se utilizó el software Primer v.6 (Clarke y Gorley 2006).

\section{Resultados}

Abundancia relativa de $G$. bogotensis: Se capturaron en total 675 individuos de G. bogotensis. El río Lenguazaque presentó la mayor abundancia (127 individuos), seguido por la laguna de Fúquene (122 individuos), río Ubaté (92 individuos) y lago de Tota A (89 individuos). En las restantes seis localidades se capturaron entre 15 y 78 individuos (Tabla 2). Cuando se compara la abundancia relativa de $G$. bogotensis por cuenca hidrográfica, en el río Bogotá fue significativamente menor que en el río Suarez (Kruskal-Wallis $=4,08 ; \mathrm{p}=0,043$ ). La CPUE en la cuenca del río Suárez fue en promedio de 103 individuos mientras que en el río Bogotá fue de 34. En el lago de Tota se presentó una CPUE promedio de 64 individuos y valores semejantes a los de las cuencas Bogotá y Suárez (Tabla 2).

Tabla 2. Captura por unidad de esfuerzo (CPUE) de Grundulus bogotensis para un esfuerzo de captura de 60 minutos en cada localidad de muestreo, en el altiplano Cundiboyacense, Colombia.

\begin{tabular}{llcc}
\hline Cuenca & Localidad & $\begin{array}{c}\text { CPUE } \\
\text { (Localidad) }\end{array}$ & $\begin{array}{c}\text { CPUE } \\
\text { (Cuenca) }\end{array}$ \\
\hline Bogotáa & Río Bogotá (Tibitoc) & 12 & \\
& Quebrada La Susana & 78 & $34 \pm 30$ \\
& Quebrada Mancilla & 15 & \\
& Embalse de Tominé & 31 & \\
Suárez & Laguna de Fúquene & 122 & \\
& Río Ubaté & 92 & \multirow{10}{*}{$102,8 \pm 26.8$} \\
& Laguna de Cucunubá & 70 & \\
& Río Lenguazaque & 127 & \\
Tota & Lago de Tota A & 89 & $64 \pm 35.2$ \\
& Lago de Tota B & 39 & \\
\hline
\end{tabular}

Dieta: En el análisis de la dieta se identificaron 30 ítems alimentares (agrupados en nueve categorías alimentarias). En general, los recursos consumidos por la especie en las tres cuencas fueron de origen autóctono. Los grupos más abundantes en la dieta fueron los insectos -Diptera (larvas y pupas de 
Chironomidae), Trichoptera y Odonata (Aeshnidae)y los microcrustáceos -Amphipoda (Hyalella sp.), Cladocera (Daphniidae) y Copepoda (Cyclopoida) (Figura 2).
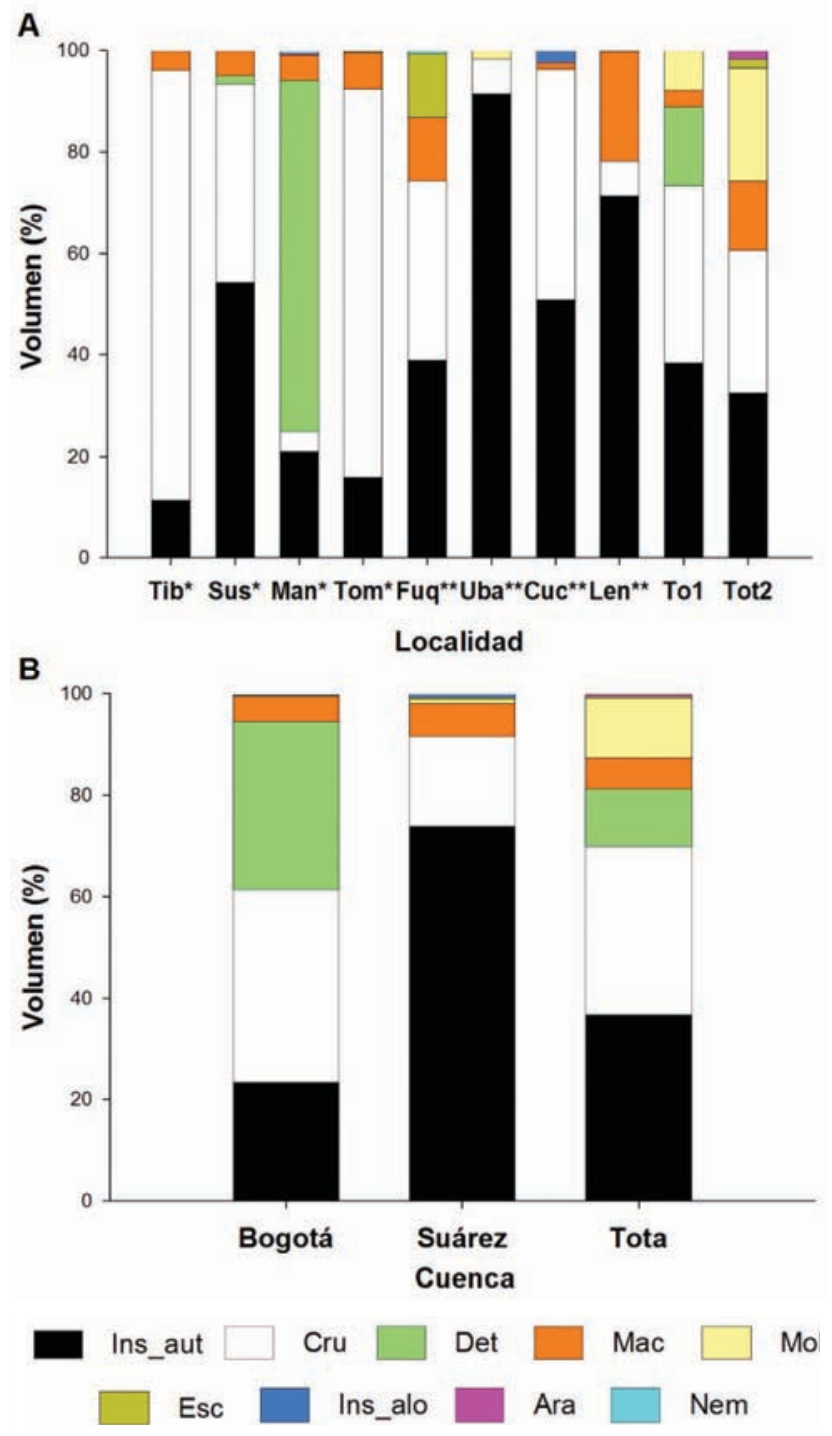

Fig. 2. Contribución porcentual de los ítems de alimento para la dieta de G. bogotensis por localidad (A) y por cuenca (B) en el altiplano Cundiboyacense, Colombia. Ítems: Ins_aut: insectos de origen autóctono; Cru: crustáceos; Det: detrito; Mac: macrófitas; Mol: moluscos; Esc: escamas; Ins_alo: insectos de origen alóctono; Ara: arácnidos de origen alóctono; Nem: nemátodos. Localidades: Tib: Tibitoc; Sus: Quebrada La Susana; Man: Quebrada Mancilla; Tom: Embalse de Tominé; Fuq: Laguna de Fúquene; Uba: Río Ubaté; Cuc: Laguna de Cucunubá; Len: Río Lenguazaque; To1: Lago de Tota A; Tot2: Lago de Tota B. Localidades (2A) marcadas con: $*$ = cuenca del río Bogotá, $* *=$ cuenca del río Suárez, sin $=$ Lago de Tota.
La gráfica del análisis NMDS (stress $=0,12$ ) indicó que no hubo una diferenciación en la dieta de $G$. bogotensis entre las tres cuencas estudiadas (Figura 3).

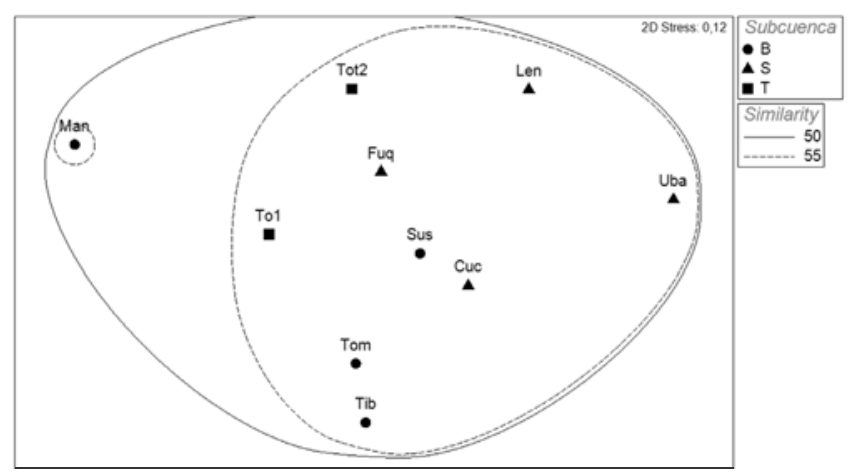

Fig. 3. Representación de la dieta de G. bogotensis por localidad y cuencas mediante el análisis de escalonamiento multidimensional no métrico (NMDS) con sobreposición de cluster (UPGMA). Cuencas (B: Bogotá; S: Suárez; T: Tota); Localidades (Tib: Tibitoc; Sus: Quebrada La Susana; Man: Quebrada Mancilla; Tom: Embalse de Tominé; Fuq: Laguna de Fúquene; Uba: Río Ubaté; Cuc: Laguna de Cucunubá; Len: Río Lenguazaque; To1: Lago de Tota A; Tot2: Lago de Tota B).

Los valores de $\mathrm{R}$ global y $\mathrm{R}$ por factor (obtenidos con el ANOSIM) no fueron significativos pero en general presentaron valores bajos $(\leq 0.321)$ (Tabla 3 ).

Tabla 3. Resultados del análisis de similaridad (ANOSIM, 999 permutaciones) de la dieta de G. bogotensis entre las tres cuencas de estudio en el altiplano Cundiboyacense, Colombia.

\begin{tabular}{lcccc}
\hline Factor & $\mathrm{R}$ (global) & $\mathrm{p}$ (global) & $\mathrm{R}$ & $\mathrm{p}$ \\
\hline Bogotá-Suárez & & & 0,219 & 0,14 \\
Bogotá-Tota & 0,221 & 0,09 & 0,107 & 0,47 \\
Suárez-Tota & & & 0,321 & 0,13 \\
\hline
\end{tabular}

Los dos análisis indicarían que la dieta entre cuencas no es exactamente la misma pero que sí difiere poco entre cuencas. En el mismo sentido, el análisis de porcentaje de similaridad (SIMPER) indicó una alta similaridad de la dieta entre y dentro de cuencas, $>60 \%$ y $>65 \%$ respectivamente (Tabla 4 ). 
Tabla 4. Resultados del análisis de porcentaje de similaridad (SIMPER) de la dieta de G. bogotensis entre cuencas y dentro de cuencas estudiadas en el altiplano Cundiboyacense, Colombia.

\begin{tabular}{lc}
\hline & Similaridad entre cuencas $(\%)$ \\
\hline Bogotá-Suárez & 60,6 \\
Bogotá-Tota & 64,1 \\
Suárez-Tota & 64,0 \\
& Similaridad dentro de cuencas (\%) \\
Bogotá & 65,1 \\
Suárez & 66,7 \\
Tota & 73,1 \\
\hline
\end{tabular}

\section{Discusión}

Abundancia relativa de $G$. bogotensis: En este estudio la abundancia relativa de $G$. bogotensis en la cuenca del río Bogotá fue significativamente menor que en la del río Suárez, resultado que podría reflejar el actual grado de deterioro de la primera. Para el año 2002, en la cuenca del río Bogotá existían 483 vertimientos de origen municipal, 672 de índole agropecuario y 2822 de tipo industrial, siendo el cuerpo de agua más contaminado de Colombia (CAR 2002) y donde las concentraciones de metales pesados exhiben valores por encima de los niveles máximos permitidos (Mancera-Rodríguez y Álvarez-León 2006). Aunque la interrelación entre aspectos de la dinámica de poblaciones, suceso en el reclutamiento y sobrevivencia debería ser entendida antes de sugerir que las diferencias en las abundancias de las poblaciones de $G$. bogotensis se deben a la contaminación de los cuerpos de agua (Elliot et al. 2007), hay evidencia de los efectos de este factor sobre algunos parámetros poblacionales de la ictiofauna. Por ejemplo, juveniles de trucha común (Salmo trutta) presentan diferencias en la tasas de mortalidad, desarrollo y crecimiento y en la proporción de malformaciones en función del gradiente de contaminación, siendo que en ambientes contaminados con químicos orgánicos y metales pesados se presenta un desarrollo retardado, reducción en la tasa de crecimiento y una alta tasa de mortalidad (Luckenbach et al. 2001).

Los valores de abundancia relativa indicarían que en la cuenca del río Suárez se presenta una mayor población de $G$. bogotensis en comparación con las otras dos cuencas. Sin embargo, se sabe que la laguna de Fúquene y sistemas lagunares asociados son cuerpos de agua eutróficos, donde el vertimiento directo de aguas residuales domésticas e industriales, la agricultura, la ganadería y en menor intensidad la disposición inadecuada de residuos sólidos domésticos son las actividades humanas que contribuyen a su deterioro (CAR 2006, RiveraRondón et al. 2008). Para el lago de Tota, Mora et al. (1992) afirman que la especie es abundante, pero se carece de un método cuantitativo de estimación que permita realizar comparaciones. Aunque el esfuerzo de muestreo en este cuerpo de agua fue menor, se debe resaltar que la CPUE fue superior que en la cuenca del río Bogotá (64 vs. 34). G. bogotensis fue trasplantada en este lago en la década de los 50's con fines piscícolas (Alvarado y Gutiérrez 2002), por lo que los resultados del presente estudio indicarían condiciones favorables para la sobrevivencia de la especie en este cuerpo de agua.

Dieta: En el presente estudio, los grupos más abundantes en la dieta de $G$. bogotensis fueron los insectos de origen autóctono y los microcrustáceos, resultado que coincide con lo reportado previamente por otros autores (Mora et al. 1992, PantojaAgreda y Pantoja-Agreda 2003, Pinzón-González y Prada-Pedreros 2011). La captura de Grundulus bogotensis en zonas con abundante presencia de macrófitas acuáticas, principalmente buchón de agua (Eichhornia sp.), elodea (Elodea sp.) y junco (Schoenoplectus californicus) coincide con lo reportado por Rivera-Rondón et al. (2008), y este factor probablemente estaría determinando el origen del recurso consumido por esta especie. En otros peces de pequeño porte pertenecientes a la familia Characidae y que habitan en zonas con presencia de macrófitas, también se ha encontrado que su dieta se compone principalmente de ítems autóctonos, representados por microcrustáceos e insectos inmaduros del orden Diptera (Casatti et al. 2003, Güntzel et al. 2012).

En este sentido, ítems que fueron consumidos por G. bogotensis, como anfípodos, Chironomidae, 
odonatos y otras larvas de insectos, son abundantes en los tallos y raíces de macrófitas sumergidas (Rozas y Odum 1988). Así mismo, la abundancia de algunos géneros de cladóceros Daphniidae y Chydoridae ha mostrado una positiva asociación con la abundancia y cobertura de macrófitas (Stansfield et al. 1997, Güntzel et al. 2010, Güntzel et al. 2012), que está relacionada con la utilización de la vegetación como refugio (Stansfield et al. 1997).

La composición de la dieta de la Guapucha también permite inferir aspectos de su distribución espacial y explotación de los recursos para alimento. $G$. bogotensis consumió presas que se localizaron tanto en el fondo como en la zona superior de la columna de agua. La presencia de granos de arena y detrito junto con larvas bentónicas de insectos (Chironomidae, Ceratopogonidae) se considera un indicador de que el alimento se está tomando del fondo (Casatti et al. 2003). Mientras que el consumo de presas que son típicamente pelágicas, como cladóceros Daphniidae (Stansfield et al. 1997), sugieren que la especie también explora en sentido ascendente la columna de agua. Sobre la distribución espacial y utilización de toda la columna de agua por parte de $G$. bogotensis ya se ha hecho referencia, indicando que la permanencia de la especie en las zonas más profundas en ecosistemas como la laguna de Fúquene, estaría restringida a intervalos cortos de tiempo debido a los bajos niveles de oxígeno (Rivera-Rondón et al. 2008).

La similaridad en la dieta de G. bogotensis en los diferentes subsistemas podría estar relacionada con la abundancia y disponibilidad de insectos de origen autóctono y microcrustáceos en los ambientes estudiados. Rivera-Rondón et al. (2008) encontraron que en los micro-hábitats donde fueron capturados ejemplares de $G$. bogotensis, los organismos dominantes eran dípteros (Chironomidae), anfípodos (Hyalella sp.), así como otros insectos de origen autóctono y microcrustáceos. En el mismo sentido, el estado trófico similar de los sistemas acuáticos estudiados probablemente influenció la similaridad de la dieta de $G$. bogotensis entre cuencas. Las condiciones tróficas de los sistemas acuáticos pueden favorecer la presencia de ciertos grupos de organismos, especialmente Chironomidae (Diptera), que tienden a ser dominantes en sistemas eutróficos con descargas de materia orgánica y bajas concentraciones de oxígeno (Fagundes y Shimizu 1997, Marques et al. 1998, Morais et al. 2010).

\section{Conclusión}

La abundancia relativa de las poblaciones de $G$. bogotensis en la cuenca del río Bogotá fue significativamente menor que en la cuenca del río Suárez. Los resultados sugieren que los sistemas acuáticos de la cuenca del río Suárez probablemente soportan la mayor abundancia de esta especie en todo su rango de distribución. Los datos también indican condiciones favorables para la sobrevivencia de la Guapucha en el lago de Tota. El análisis de la dieta indicó que la Guapucha es una especie consumidora de ítems de origen autóctono (insectos y microcrustáceos) que exhibe una dieta similar en las tres cuencas estudiadas.

\section{Agradecimientos}

Este estudio se desarrolló en el marco del proyecto: "Variación morfológica y abundancia relativa de poblaciones de Grundulus bogotensis (Humboldt, 1821) (Characiformes: Characidae), en el altiplano Cundiboyacense, Colombia", financiado por la Pontificia Universidad Javeriana (Proyecto $\mathrm{N}^{\circ} 287$ ) y el Instituto de Investigación de Recursos Biológicos Alexander von Humboldt. Expresamos nuestro agradecimiento a la Vicerrectoría Académica de la Pontificia Universidad Javeriana y al Instituto de Investigación de Recursos Biológicos Alexander von Humboldt por la financiación de este trabajo; a los profesores Enrique Forero Useche, Andrés R. Acosta Galvis y a los revisores anónimos por la lectura crítica del manuscrito en su versión inicial; a los miembros del laboratorio de ictiología de la Pontificia Universidad Javeriana y a Javier Molina y Francisco Ciri por su colaboración en el trabajo de campo. Los autores declaran no tener conflicto de interés con relación a este trabajo.

\section{Referencias}

Agostinho AA, Thomaz SM, Gomes LC (2005) Conservation of the biodiversity of Brazil's inland waters. Conservation Biology 19(3): 646-652

Albrecht PA, Caramaschi EP (2003) Feeding ecology of Leporinus friderici (Teleostei; Anostomidae) in the Uper Tocantins River, Central Brazil, before and 
after installation of a hydroelectric plant. Studies on Neotropical Fauna and Environment 38(1): 33-40.

Alvarado H, Gutiérrez FP (2002) Especies hidrobiológicas continentales introducidas y trasplantadas y su distribución en Colombia. Ministerio de Medio Ambiente - Instituto de Investigaciones Alexander von Humboldt. Bogotá D.C., 167p

Álvarez-León R, González JA, Forero JE (2002) Grundulus bogotensis. En: Mojica JI, Castellanos C, Usma S, Álvarez R, editores. Libro rojo de peces dulceacuícolas de Colombia. La serie Libros Rojos de Especies Amenazadas de Colombia. Instituto de Ciencias Naturales, Universidad Nacional de Colombia, Ministerio del Medio Ambiente. Bogotá, Colombia, pp. 200-202

Álvarez-León R, Hernández-Barrero S, GonzálezAcosta JA, Valderrama M, Forero-Useche JE, et al. (2012) Grundulus bogotensis. En: Mojica JI, Usma JS, Álvarez-León R, Lasso CA, editores. Libro rojo de peces dulceacuícolas de Colombia. Instituto de Investigación de Recursos Biológicos Alexander von Humboldt, Instituto de Ciencias Naturales de la Universidad Nacional de Colombia, WWF Colombia y Universidad de Manizales. Bogotá, D.C., Colombia, pp. 239-242

Battaile BC, Quinn II TJ (2004) Catch per unit effort standardization of the eastern Bering Sea walleye pollock (Theragra chalcogramma) fleet. Fisheries Research 70: 161-177

Brown JH (1984) On the relationship between abundance and distribution of species. The American Naturalist 124(2): 255-279

CAR (2002) Atlas Ambiental CAR 2001. Primera edición. Corporación Autónoma Regional de Cundinamarca. Bogotá, Colombia, $175 \mathrm{p}$

CAR (2006) Diagnóstico prospectiva y formulación de la cuenca hidrográfica de los ríos Ubaté y Suárez - Plan de Ordenamiento de la Cuenca de los Ríos Ubaté y Suárez. Informe general. Corporación Autónoma Regional de Cundinamarca. Bogotá, Colombia, 289 p

Casatti L, Mendes HF, Ferreira KM (2003) Aquatic macrophytes as feeding site for small fishes in the Rosana reservoir, Paranapanema river, southeastern Brazil. Brazilian Journal of Biology 63(2): 213-222

Clarke KR, Gorley RN (2006) PRIMER v6: user manual/ tutorial. Plymouth: PRIMER-E

Collares-Pereira MJ, Cowx IG, Ribeiro F, Rodrigues JÁ, Rogado L (2000) Threats imposed by water resource development schemes on the conservation of endangered fish species in the Guadiana River Basin in Portugal. Fisheries Management and Ecology 7: 167-178

Dahl G (1971) Los peces del norte de Colombia. Ministerio de Agricultura, Instituto de Desarrollo de los Recursos Naturales Renovables - INDERENA. Bogotá, Colombia, 390 p

Elliott M, Hemingway KL, Krueger D, Thiel R, Hylland
K, Arukwe A, Förlin L, Sayer M (2007) From the individual to the population and community responses to pollution. En: Lawrence A, Hemingway K, editors. Effects of pollution on fish: molecular effects and population responses. Blackwell Science Ltd. Oxford, UK, pp. 221-255. doi: 10.1002/9780470999691.ch6

Fagundes RC, Shimizu GY (1997) Avaliação da qualidade da água do Rio Sorocaba-SP, através da comunidade bentônica. Revista Brasileira de Ecologia 1: 63-66

Fowler HW (1942) Lista de peces de Colombia. Revista de La Academia Colombiana de Ciencias Exactas, Físicas y Naturales 5(17): 128-138

Gorman OT, Karr JR (1978) Habitat structure and stream fish communities. Ecology 59: 507-515

González-Acosta JA, Bueno M, Forero-Useche JE (1992) Caracterización cromosómica de dos especies ícticas nativas: Guapucha, (Grundulus bogotensis) y Capitán, (Eremophilus mutisiz), de la sabana de Bogotá. Acta Biológica Colombiana 7-8: 45-54

Güntzel AM, Panarelli EA, da Silva WM, Roche KF (2010) Influence of connectivity on Cladocera diversity in oxbow lakes in the Taquari River floodplain (MS, Brazil). Acta Limnologica Brasiliensia 22(1): 93-101. http://dx.doi.org/10.4322/actalb.02201012

Güntzel AM, Melo IK, Roche KF, da Silva VFB, Pompiani PG (2012) Cladocerans from gut contents of fishes associated to macrophytes from Taquari River Basin, MS, Brazil. Acta Limnologica Brasiliensia 24(1): 97-102. http://dx.doi.org/10.1590/S2179975X2012005000029

Haggarty DR, King JR (2006) CPUE as an index of relative abundance for nearshore reef fishes. Fisheries Research 81(1): 89-93

Harley SJ, Myers RA, Dunn A (2001) Is catch-per-uniteffort proportional to abundance? Canadian Journal of Fisheries and Aquatic Sciences 58(9): 1760-1772

IGAC (1984) Estudio regional integrado del altiplano Cundiboyacense. Proyecto IGAC-ORSTOM. Bogotá, Colombia, $498 \mathrm{p}$

Kruk A (2007) Role of habitat degradation in determining fish distribution and abundance along the lowland Warta River, Poland. Journal of Applied Ichthyology 23: 9-18

León LD, González JA, Forero JE (2005) Aspectos biométricos de la Guapucha, Grundulus bogotensis (Pisces: Characidae). Dablia 8: 71-77

Lizama MAP, Ambrósio AM (2002) Condition factor in nine species of fish of the Characidae family in the upper Paraná river floodplain, Brazil. Brazilian Journal of Biology 62 (1): 113-124

Lobón-Cerviá J (1991) Dinámica de poblaciones de peces en ríos. Pesca eléctrica y métodos de capturas sucesivas en la estima de abundancias. Monografías del Museo Nacional de Ciencias Naturales. Centro de Investigaciones de Agua (C.S.I.C.). Madrid, España, $156 \mathrm{p}$ 
Luckenbach T, Triebskorn R, Müller E, Oberemm A (2001) Toxicity of waters from two streams to early life stages of brown trout (Salmo trutta f. fario L.), tested under semi-field conditions. Chemosphere 45: 571-579

Magurran AE, Phillip DAT (2001) Implications of species loss in freshwater fish assemblages. Ecography 24: 645-650

Mancera-Rodríguez NJ, Álvarez-León R (2006) Estado del conocimiento de las concentraciones de mercurio y otros metales pesados en peces dulceacuícolas de Colombia. Acta Biológica Colombiana 11 (1): 3-23

Marques MM, Barbosa FAR, Callisto M (1998) Distribution and abundance of Chironomidae (Diptera, Insecta) in an impacted watershed in southeast Brazil. Revista Brasileira de Biologia 59 (4): 553-561

McCafferty WP, Provonsha AV (1981) Aquatic entomology. Science Books Internacional, Boston, $448 \mathrm{p}$

Merritt RW, Cummins KW (1984) An introduction to the aquatic insects of North America. Second Edition. Kendall/Hunt Publishing Co. Dubuque, Iowa, $722 \mathrm{p}$

Miles C (1971) Los peces del río Magdalena. Segunda Edición, Universidad del Tolima, Ibagué, Colombia, $214 \mathrm{p}$

Mojica JI, Castellanos C, Usma S, Álvarez-León R (Eds.) (2002) Libro rojo de peces dulceacuícolas de Colombia. Instituto de Ciencias Naturales, Universidad Nacional de Colombia, Ministerio del Medio Ambiente. Bogotá, Colombia, $288 \mathrm{p}$

Mojica, JI, Usma JS, Álvarez-León R, Lasso CA (Eds) (2012) Libro rojo de peces dulceacuícolas de Colombia - 2012. Instituto de Investigación de Recursos Biológicos Alexander von Humboldt, Instituto de Ciencias Naturales de la Universidad Nacional de Colombia, WWF Colombia y Universidad de Manizales. Bogotá, Colombia, 319 p

Mora G, Téllez LS, Cala P, Guillot G (1992) Estudio bioecológico de la ictiofauna del lago de Tota (Boyacá-Colombia), con énfasis en la trucha arco iris, Oncorbynchus mykiss. Revista de la Academia Colombiana de Ciencias Exactas, Físicas y Naturales 18 (70): 409-422

Morais SS, Molozzi JI, Viana AL, Viana TH, Callisto M (2010) Diversity of larvae of littoral Chironomidae (Diptera: Insecta) and their role as bioindicators in urban reservoirs of different trophic levels. Brazilian Journal of Biology 70(4): 995-1004. http://dx.doi. org/10.1590/S1519-69842010000500011

Pantoja-Agreda F, Pantoja-Agreda C (2003) Hábitos alimentarios de Grundulus sp. (Pisces: Characidae) em el lago Guamuéz, Departamento de Nariño, Colombia. Dablia 6: 113-115

Pinzón-González AM, Prada-Pedreros S (2011) Composición de la dieta de la Guapucha, Grundulus bogotensis (Osteichthyes: Characidae), en la laguna de Fúquene, altiplano Cundiboyacense, Colombia. Dablia 11: 43-52

Rivera-Rondón C, Prada-Pedreros S, Galindo D, Maldonado-Ocampo JA (2008) Effects of aquatic vegetation on the spatial distribution of Grundulus bogotensis, Humboldt 1821 (Characiformes: Characidae). Caldasia 30 (1): 135-150

Rocha FC da, Casatti L, Pereira DC (2009) Structure and feeding of a stream fish assemblage in Southeastern Brazil: evidence of low seasonal influences. Acta Limnologica Brasiliensia 21 (1): 123-134

Roldán G (1996) Guía para el estudio de los macroinvertebrados acuáticos del departamento de Antioquia. Universidad de Antioquia, Facultad de Ciencias Exactas y Naturales. Centro de Investigaciones - CIEN, Medellín, Colombia. Fondo FEN Colombia, COLCIENCIAS, Bogotá, Colombia, 217 p

Rozas LP, Odum WE (1988) Occupation of submerged aquatic vegetation by fishes: testing the roles of food and refuge. Oecologia 77: 101-106

Stansfield JH, Perrow MR, Tench LD, Jowitt AJD, Taylor AAL (1997) Submerged macrophytes as refuges for grazing Cladocera against fish predation: observations on seasonal changes in relation to macrophyte cover and predation pressure. Hydrobiologia 342/343: 229240

StatSoft, Inc. (2004) STATISTICA (data analysis software system), version 7. www.statsoft.com

Tejerina-Garro FL, Maldonado M, Ibáñez C, Pont D, Roset N, Oberdorff T (2005) Effects of natural and anthropogenic environmental changes on riverine fish assemblages: a framework for ecological assessment of rivers. Brazilian Archives of Biology and Technology 48: 91-108 